Renal cell carcinoma (RCC) comprises 3-4\% of all malignant tumours among adults in Poland. Spontaneous regression of RCC is a rare but well-known phenomenon. Its frequency is estimated to be approximately $1 \%$ and a large part of the percentage is accounted for by the regression of pulmonary metastases in the course of clear type of RCC treatment. We searched PubMed, Embase and SciVerse Scopus databases, identifying 59 case reports of spontaneous regression of RCC. Those medical histories come from reports from around the world and date back up to 40 years. This review includes their analysis as well as description of possible explanations of this phenomenon postulated by different authors, including both misdiagnosis and immunological reactions. This study indicates that reliable diagnostics and reporting of all the cases of spontaneous regression play a key role, as this is the only method which enables a better perspective in understanding this issue.

Key words: renal cell carcinoma, neoplasm regression, kidney cancer, metastasis, spontaneous remission.

\section{Spontaneous regression of renal cell carcinoma}

\section{Anna Daria Janiszewska, Sławomir Poletajew, Aleksander Wasiutyński}

Department of Pathology, Medical University of Warsaw, Warsaw, Poland

\section{Introduction}

The phenomenon of spontaneous regression of cancer was described by many authors in relation to many types of neoplasms over the last century $[1,2]$. One of the most frequently reported malignancies in those reviews was renal cell carcinoma (RCC). Renal cell carcinoma makes up 3-4\% of all malignant tumours among adults in Poland. The dominant subtype is clear cell RCC, whose occurrence is estimated to represent more than $85 \%$ of all RCC cases [3]. According to many reviews describing spontaneous regression, RCC is one of the most frequent types of malignant neoplasm related to this phenomenon. The rate of spontaneous regression of RCC versus other malignancies is estimated to be several times higher [4]. The frequency of spontaneous regression in RCC patients is estimated to be approximately $1 \%$ [5]. Since the first publication by Bumpus in 1928, many authors have tried to analyse and explain this phenomenon. Each year new cases of spontaneous regression of RCC are documented in the literature worldwide. We analysed over 50 medical histories dating back up to 40 years and gathered information that would possibly explain this phenomenon.

\section{Spontaneous regression}

Spontaneous regression is defined as the partial or complete disappearance of a tumour without any treatment or with treatment which is generally perceived as irrelevant to the progression of cancer [1]. It is important to emphasize that this definition does not necessarily mean a complete cure of the cancer, but rather the inhibition of the progression or disappearance of metastases. The number of cases of spontaneous regression in RCC is estimated to be between $0.4 \%$ and $4 \%$, but in the majority of publications it is approximated to $1 \%$ of all the cases of renal cancer [6]. Many of the authors classify regression into at least 4 types of spontaneous regression: regression of primary tumour, regression of metastases (with or without pathological confirmation), prolonged remission, and delayed metastases [1]. The number of cases in each type differs significantly. The majority of cases concern regression of metastases, whereas regression of primary tumour and delayed metastases are small groups with several cases (Table 1). We have not found any report on prolonged remission of RCC. Almost all reported cases concern clear cell RCC with a few exceptions. Unfortunately, not all the cases had sufficiently extensive follow-up; it varies between 1 year and 16 years.

\section{Regression of metastatic renal cell carcinoma}

Among all RCC cases, metastasis occurs in about a third of them, with the overall survival not exceeding 12 months when untreated, while less then $10 \%$ of these cases have a 5-year survival [7]. Regression of metastatic sites included mainly the lungs, then: the liver, the bones, the adrenal glands, the pleura, the brain, the choroid, and the pancreas (Fig. 1). Detailed data are presented in Table 1 . The majority of metastases regressed spontaneously after nephrectomy or embolization of the primary tumour. The interval between 
Table 1. Summary of all reported cases of spontaneous regression of RCC from 1971 to 2011

\begin{tabular}{|lccc}
\hline $\begin{array}{l}\text { Type of regression } \\
\text { Regression of primary }\end{array}$ & Organs & $\begin{array}{c}\text { Number } \\
\text { of reports }\end{array}$ & $\begin{array}{c}\text { Number } \\
\text { of cases }\end{array}$ \\
tumour & kidney & 4 & 5 \\
Delayed metastases & lungs & 5 & 5 \\
& pancreas & 1 & 1 \\
Regression of & lungs & 27 & 30 \\
metastases & lungs, adrenal gland & 1 & 1 \\
& lungs, bones & 1 & 1 \\
& lungs, brain & 1 & 1 \\
& lungs, pleura & 1 & 1 \\
& lungs, scalp & 1 & 1 \\
& liver & 4 & 4 \\
& bones & 2 & 2 \\
& bones, liver & 1 & 1 \\
choroid & 2 & 2 \\
pleura & 2 & 2 \\
brain & 1 & 1 \\
pancreas & 1 & 1 \\
total & 55 & 59 \\
& &
\end{tabular}

nephrectomy and noticeable regression differs among all cases - it followed after days, weeks, months or even a year. It is important to emphasize that metastases from RCC were diagnosed either by histological examination or they were presumed radiologically. Only some of the patients received steroids or hormone therapy, but this kind of treatment is perceived as palliative. Recently there have been reported cases of metastasis regression after a short course of treat-

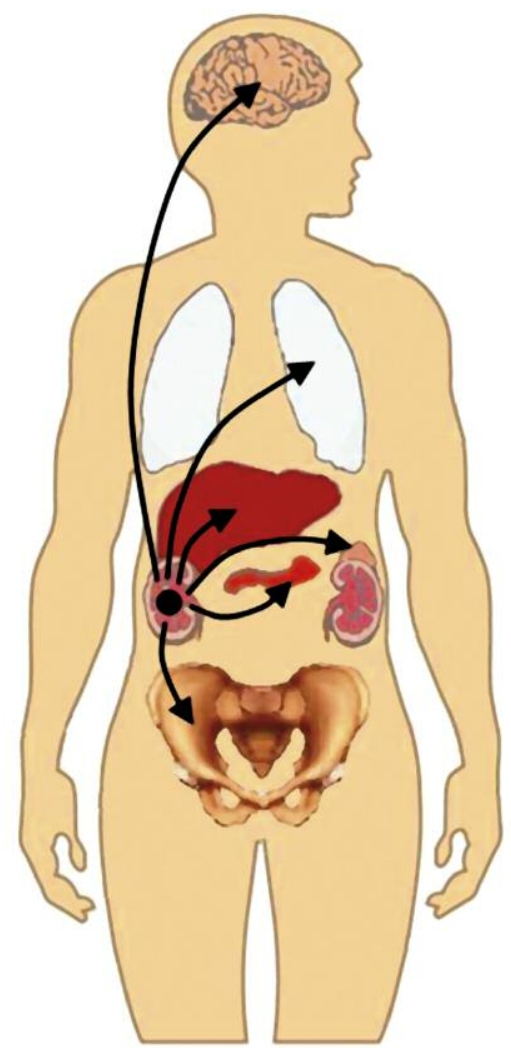

Fig. 1. The most frequently affected organs in metastatic RCC ment with sunitinib (multi-targeted receptor tyrosine kinase inhibitor).

\section{Regression of primary renal tumour}

The reported cases of regression of primary tumour were associated either with previous haemorrhage into tumour [8] or with renal vein emboli [9]. The lesions observed in the tumours on pathological examination which have spontaneously regressed were usually: fibrosis, hyalinization, calcification or ossification [10]. The diagnosis of RCC was often based on thorough sampling of the specimen and sometimes established only on the basis of immunohistochemical staining. The characteristic antigens expressed on RCC cells are keratins, epithelial mucin antibody, carcinoembryonic antigen and vimentin [11].

\section{Delayed metastases from renal cell carcinoma}

Renal cell carcinoma has a very high tendency to metastasize, with the lesions appearing shortly after an early diagnosis. Therefore delayed metastases are perceived as a kind of regression. We found 5 cases of delayed pulmonary metastases which occurred from 9 to 300 months after the diagnosis [12-16]. There was also a history of 20 years delayed pancreatic metastasis in this group [17]. All cases were preceded by nephrectomy and most of the metastases were located in lungs. The pathological confirmation of the tumour characteristics was obtained in each case. Most of the lesions were detected accidentally during the postoperative follow-up.

\section{True or illusion?}

Despite the fact that cases of spontaneous regression of RCC have been reported from over the last 80 years, it is important to consider all the uncertainties associated with this phenomenon. Keilholz divides these factors into 3:

- related to wrong diagnosis;

- related to lack of histological confirmation;

- related to incorrect interpretation of simultaneous changes in the patients' condition [18].

In most reviews considering this issue, these problems are mentioned. A majority of cases of spontaneous regression pertain to pulmonary metastases - which without histological confirmation might be illusive. Pulmonary infarcts caused by e.g. emboli may mimic metastases in lungs on radiographic scans, a fact that can lead to a false conclusion of the disappearance of cancer [19].

\section{Lack of reliable data}

There are also other noticeable difficulties with reporting the phenomenon of spontaneous regression. Hobohm emphasizes the impossibility of describing cases of regression due to the physician's unawareness or unwillingness. He also blames a situation when a patient does not come for follow-up visits, causing lack of his medical history [20]. The subclinical stage of disease may also be responsible for some of the cases of spontaneous regression, especially those associated with delayed metastases. Moreover, the number of reported cases is insufficient for the conduction of any 
detailed survey or the identification of any definite explanations of this phenomenon. Most of the reports do not include any kind of interpretation of spontaneous regression.

\section{Genetic factors}

The fact that the mutation of the von Hippel Lindau (VHL) gene pertains to the majority of cases of RCC is well known. Mutations of the VHL tumour suppressor gene occurred both in sporadic RCC as well as in a hereditary cancer syndrome. This genetic change leads to increased production of vascular endothelial growth factor, which results in a higher tendency of cancer invasion. It is impossible to decide which genetic or epigenetic factors are associated with the phenomenon of spontaneous regression. Unfortunately, the lack of complex molecular analysis of regressed tumours makes the investigation difficult. The postulated molecular mechanism is apparently linked to the process of apoptosis induced by different cellular or extracellular changes.

\section{Evidence and hypotheses}

A majority of cases of spontaneous regression are associated with kidney resection. Undoubtedly, nephrectomy is the gold standard treatment for RCC and plays an important role in the induction of regression. The same is mentioned in the guidelines of the European Organisation for Research and Treatment of Cancer Genito-Urinary Tract Cancer Group from 2009, it has also been noted that: "Nephrectomy is an important component of the multimodality treatment of metastatic RCC. This procedure induces spontaneous regression of metastases in a small number of patients [Level 4]" [21]. However, in a majority of articles, spontaneous regression is perceived as not necessarily related to nephrectomy [22]. The resection of the primary tumour results in elimination of substances secreted by the neoplasm (i.e. growth or antiapoptotic factors) and suppresses the progression of cancer. Moreover, the removal of the cancer enables the immune system to defend the organism by depletion of the load of cancer antigens [23]. The dissemination of tumour cells which may occur in the course of resection might initiate higher antigen exposure, which could result in an antitumour response of the immune system [6].

Since the appearance of the first reported case of spontaneous regression of RCC, the immune mechanism was postulated as being responsible for regression. Riese et al. distinguished various aetiological factors, which include:

- unexpected self-healing host mechanism,

- changes induced by hormone alterations or by trauma (e.g. fever, infection, surgery) [6].

The same factors are mentioned in other studies on this issue. The link between spontaneous regression of the tumour and the response to the treatment with cytokines is noticeable not only in RCC cases but also in malignant melanomas. Many authors accentuate the role of inflammation caused by bacterial infections or the role of non-specific inflammatory stimuli. It seems important to mention that several dozen patients who experienced spontaneous regression of cancer had an incidence of a major feverish infection during the course of the disease [20]. It should be taken into consideration that activation of the immune system by various provocative stimuli can result in overcoming the condition of tumour tolerance. It has been observed that in the case of the simultaneous occurrence of RCC and autoimmunological disease, the regression of RCC is accompanied by the worsening of the latter. This can be shown by a case where a patient with psoriasis and metastatic RCC underwent nephrectomy after which a spontaneous regression of pulmonary metas-
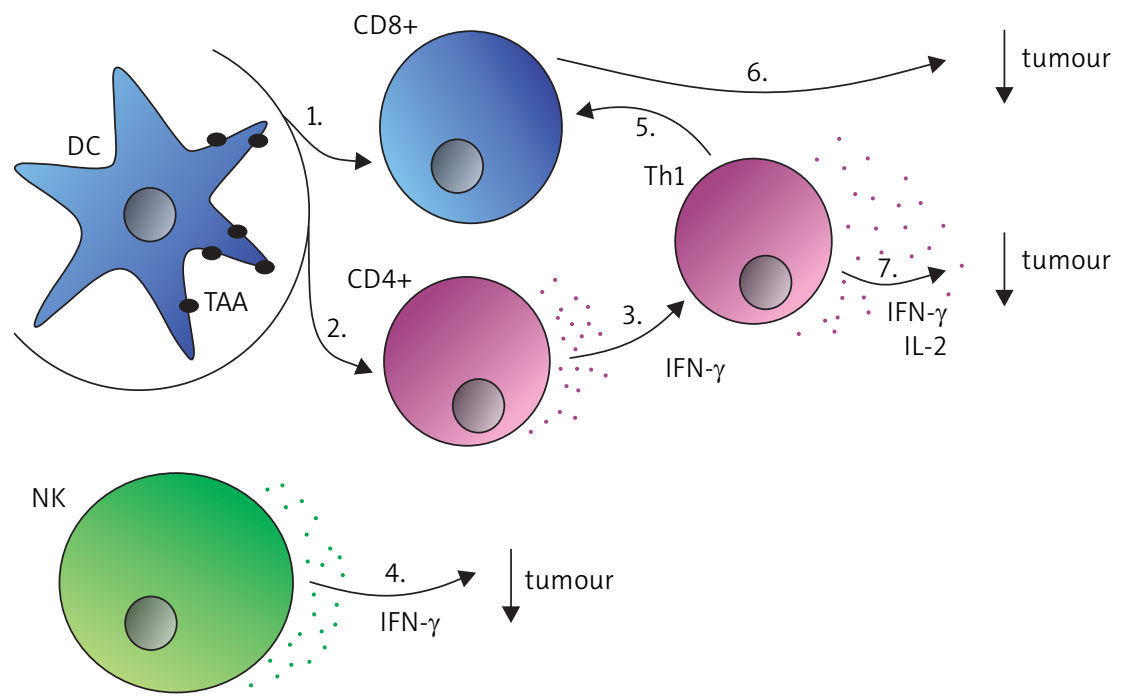

DC presenting TAA migrates to the lymph nodes where it interacts with Tlymphocytes, activating CD8+ (1) and CD4+ (2). Proliferating CD4+ cells develop into effector T cells - Th1 (3). NK secreting IFN- $\gamma$ activate cytolytic effector cells, which promote the expression of Fas ligand, which induces apoptosis of tumour cells (4). Th1 activating CD8+ (5). CD8+ destroys tumour cells (6). Th1 releases IFN- $\gamma$ and IL-2 which mobilizes the immune effector cells (7).

DC-dendritic cell, CD8+-CD8+Tlymphocyte, CD4+-CD4+Tlymphocyte, Th1 - Thelper cell type 1, NK-natural killer cells, TAA-tumour associated antigens, IFN- $\gamma$ - interferon- $\gamma$, IL-2 - interleukin-2

Fig. 2. The role of the immune response in tumour rejection 
tases was observed simultaneously with the exacerbation of psoriasis [24]. The incidence of the intensification of the autoimmune disease (triggered by infection or other factors) may confirm the linkage between the spontaneous cancer regression and activation of the immune system.

\section{Role of cytokine therapy}

The last 5 years have completely changed the pharmacotherapy of metastatic RCC with the appearance of new molecular targeted drugs. Previously, cytokine therapy consisting of interleukin-2 (IL-2) and interferon- $\alpha$ (IFN- $\alpha$ ) was used. The administration of IL-2 and IFN- $\alpha$ was associated with low rates of response and high toxicity; however, the combination of those agents with new therapies is still in use. Interleukin-2 is responsible for the mobilization of immune effector cells in order to destroy cancer (Fig. 2). This cytokine causes an expansion of regulatory cells which modifies the immune response. Interferon- $\alpha$ induces expression of major histocompatibility complex molecules and tumourassociated antigens as well as enhancing the function of T-cell and dendritic cells (DC). Dendritic cells belong to antigen-presenting cells, which are able to activate an inflammatory anti-tumour reaction. Schwaab et al., who worked on intranodal autologous tumour lysate-dendritic cell vaccine with IL-2 and IFN- $\alpha 2$ a therapy, noticed that the time of clinical response for this treatment differed among patients, suggesting the variance of predispositions toward immune responsiveness [25]. It is also worth mentioning that the role of the relative increase in the number of elements of the immune system is more valuable than the absolute increase. It pertains especially to natural killer cells and CD8+ T cells [26]. All those facts and findings validate the undeniable role of the immune system in RCC regression.

\section{Psychological aspects}

Some authors have mentioned an association of a psychological mechanism with spontaneous regression of the tumour. It is impossible to exclude this explanation, but it seems plausible only if combined with the endocrine and immune system theory [2]. Measurement of the influence of psychopathological variables on the regression of cancer is nearly impossible, although there are some reports which indicate that stress inhibits cellular immune responses associated with natural killer cells and T-cells [27].

\section{Summary}

Spontaneous regression of RCC tumours is a well-known phenomenon, described by many investigators. Despite many reports and many hypotheses, at present we cannot accentuate one possible explanation for this oncological phenomenon. Reliable diagnostics and reporting of all the cases of spontaneous regression play a key role, as this is the only method which enables a better perspective in understanding this issue. Due to the new standards of treatment of metastatic RCC (nephrectomy and pharmacotherapy) it may seem impossible to investigate this problem, but new cases are still documented. A deep analysis of each case is necessary, as it would show some similarities in the cases.
The authors declare no conflict of interest.

\section{References}

1. Cole WH, Everson TC. Spontaneous Regression of Cancer: Preliminary Report. Ann Surg 1956; 144: 366-80.

2. Challis GB, Stam HJ. The spontaneous regression of cancer. A review of cases from 1900 to 1987. Acta Oncol 1990; 29: 545-50.

3. Chow WH, Devesa SS. Contemporary epidemiology of renal cell cancer. Cancer J 2008; 14: 288-301.

4. Chodorowski Z, Anand JS, Wiśniewski M, Madaliński M, Wierzba K, Wiśniewski J. Spontaneous regression of cancer - review of cases from 1988 to 2006. Przegl Lek 2007; 64: 380-2.

5. Snow RM, Schellhammer PF. Spontaneous regression of metastatic renal cell carcinoma. Urology 1982; 20: 177-81.

6. de Riese W, Goldenberg K, Allhoff E, Stief C, Schlick R, Liedke S, Jonas U. Metastatic renal cell carcinoma (RCC): spontaneous regression, long-term survival and late recurrence. Int Urol Nephrol 1991; 23: 13-25.

7. Siebels M, Schendel D. New challenges and chances in the treatment of metastatic renal cell carcinoma. World J Urol 2005; 23: 153-4.

8. Edwards MJ, Anderson JA, Angel JR, Harty JI. Spontaneous regression of primary and metastatic renal cell carcinoma. J Urol 1996; 155: 1385

9. Kobayashi K, Sato T, Sunaoshi K, Takahashi A, Tamakawa M. Spontaneous regression of primary renal cell carcinoma with inferior vena caval tumor thrombus. J Urol 2002; 167: 242-3.

10. Choi SK, Chang SK, Lee JM, Jung WH, Park Cl. Spontaneous regression of primary renal cell carcinoma - a case report. Yonsei Med J 1986; 27: 314-7.

11. Hamid Y, Poller DN. Spontaneous regression of renal cell carcinoma: a pitfall in diagnosis of renal lesions. J Clin Pathol 1998; 51: 334-6.

12. Donaldson JC, Slease RB, DuFour DR, Saltzman AR. Metastatic renal cell carcinoma 24 years after nephrectomy. JAMA 1976; 236: 950-1.

13. Kaneko M, Matsumoto S, Tahara H, Ishii T, Uemura H. Pulmonary metastasis of renal cell carcinoma 20 years after nephrectomy. Hinyokika Kiyo 2006; 52: 929-31.

14. Sasano S, Kei J, Kaguraoka H, lida H, Yoh T, Nitta S. A case of pulmonary solitary metastasis of renal cell carcinoma nine years after radical nephrectomy. Nihon Kyobu Geka Gakkai Zasshi 1993; 41: 1105-9.

15. Shiono S, Yoshida J, Nishimura M, Nitadori J, Ishii G, Nishiwaki Y, Nagai K. Late pulmonary metastasis of renal cell carcinoma resected 25 years after nephrectomy. Jpn J Clin Oncol 2004; 34: 46-9.

16. Yoshida J, Nagai K, Hasebe T, Takahashi K, Nishimura M, Otsuka S, Nishiwaki Y, Kodama T. Pulmonary metastasis of renal cell carcinoma resected sixteen years after nephrectomy. Jpn J Clin Oncol 1995; 25: $20-4$

17. Norton KS, Zibari GB. Body and distal pancreatectomy for metastatic renal cell carcinoma: case report and review of the literature. I La State Med Soc 2004; 156: 40-1.

18. Keilholz U. Disappearing cancer. Int J Colorectal Dis 2007; 22: 737-8.

19. Wagner JR, Merino MJ, Pass HI, Linehan WM, Walther MM. Pulmonary infarcts can mimic pulmonary metastases from renal cancer. J Urol 1997; 158: 1688-90.

20. Hobohm U. Fever therapy revisited. Br J Cancer 2005; 92: 421-5.

21. de Reijke TM, Bellmunt J, van Poppel H, Marreaud S, Aapro M. EORTCGU group expert opinion on metastatic renal cell cancer. Eur J Cancer 2009; 45: 765-73.

22. Chang KC, Chan KL, Lam CW. Spontaneous regression of renal cell carcinoma metastases. Hong Kong Med J 1999; 5: 72-5.

23. Lekanidi K, Vlachou PA, Morgan B, Vasanthan S. Spontaneous regression of metastatic renal cell carcinoma: case report. J Med Case Rep 2007; 18: 89.

24. Melichar B, Vanecková J, Morávek P, Urminská H, Podhola M. Spontaneous regression of renal cell carcinoma lung metastases in a patient with psoriasis. Acta Oncol 2009; 48: 925-7.

25. Schwaab T, Schwarzer A, Wolf B, et al. Clinical and immunologic effects of intranodal autologous tumor lysate-dendritic cell vac 
cine with Aldesleukin (Interleukin 2) and IFN-\{alpha\}2a therapy in metastatic renal cell carcinoma patients. Clin Cancer Res 2009; 15: 4986-92.

26. Ricci SB, Cerchiari U. Spontaneous regression of malignant tumors: Importance of the immune system and other factors (Review). Oncol Lett 2010; 1: 941-6.

27. Andersen BL, Farrar WB, Golden-Kreutz D, Kutz LA, MacCallum R, Courtney ME, Glaser R. Stress and immune responses after surgical treatment for regional breast cancer. J Natl Cancer Inst 1988; 90: 30-6.

\section{Address for correspondence}

\section{Anna D. Janiszewska}

Department of Pathology

Medical University of Warsaw

T. Chałubińskiego 5

02-004 Warsaw, Poland

e-mail: anka.janiszewska@wp.pl

Submitted: 9.08 .2012

Accepted: 21.11.2012 